\title{
There is no logical negation: true, false, both and neither
}

\author{
Jc Beall \\ entailments.net \\ University of Connecticut \\ University of Tasmania
}

\section{Unscientific preamble: Confession of a former logical exhaustivist}

I have thought hard and long about truth-theoretic paradoxes and I am convinced that they are best understood as motivating nonclassical logic. At an early stage - though mostly behind published doors - I strongly believed that the natural position on logical negation was to maintain that paradoxical phenomena motivate a rejection of both excluded middle and its dual 'explosion'. Some of this thinking showed up in early work [5], but for one reason or another (embarrassingly, peer pressure probably being part of it) I found myself sticking to just one side or the other - rejecting excluded middle or its dual explosion but not both. Eventually, when the pressures to publicly pronounce a position grew strong I wound up embracing excluded middle, landing in the vicinity of glut theorists like Florencio Asenjo [3, 4], Routley/Sylvan [44], Priest [37, 38], Dowden [17], Mortensen [33], Weber [51], Hyde [52], Colyvan [11], Berto [14] and others. But even in my principal work on the topic [6] I explicitly expressed discomfort in embracing excluded middle for logical negation while rejecting its dual. I lived with the discomfort until now.

I'm now back to my roots. And the simple reason is at bottom tied to balance, simplicity, naturalness and the like - the very things that had me rejecting both excluded middle and explosion in my youthful thinking. A return to the simplicity of youth affords not only more logical options; it affords a better philosophical picture of logic than leading 'subclassical-logic' views offer - my chief concern in this paper.

Logic is said to be subclassical if and only if every logically valid argument is classically valid but not vice versa. In this paper I advance and defend a 
very simple position according to which logic is subclassical but is weaker than the leading subclassical-logic views have it.

One salient feature of the view I advance concerns logical negation. On the target picture there is no philosophically interesting logical negation (aside from De Morgan behavior), no interesting negation connective whose stand-alone behavior (independent of interaction with other connectives) is characterized by logic itself. This is a radical departure from otherwise 'conservative' subclassical-logic positions. My aim is to show that the radical departure is not only philosophically motivated; it results in a simple and plausible view of logic.

I am not in position to argue that the proposed view is the only sensible the true - position; but I do hope that the position is clear enough to challenge its popular opponents. (Confession over.)

\section{Puzzling phenomena and logic}

Many philosophers think that puzzling phenomena motivate non-standard logic and in particular subclassical logic. I am such a philosopher. Vagueness, semantical antinomies and more suggest that logic is not as the socalled classical story of logic has it. On target views such phenomena do not motivate any classically invalid forms but only suggest that the list of classically valid forms goes too far: as far as logic is concerned there are fewer logically valid forms than classical logic demands.

This perspective is common in contemporary philosophy: namely, that a variety of puzzling phenomena - notably paradoxical or antinomic phenomena - motivate a weaker-than-classical picture of logic. The two leading candidates along these lines are Strong Kleene K3 [28] and the Logic of Paradox LP $[3,37]$. To speak figuratively, the former demands consistency with respect to logical negation while allowing for incompleteness with respect to logical negation; and the latter does the opposite - demanding completeness while allowing inconsistency.

Many of the target debates surrounding puzzling phenomena - particularly the paradoxes - revolve around the virtues and vices of the Strong Kleene approach versus the LP approach, where these are regarded, respectively, as the leading 'gap-theoretic approach' vs 'glut-theoretic approach'. (These are reviewed in $\S 3.3$ below though some familiarity with them is presupposed.)

I remain convinced that the right account of logic is one according to which logic is subclassical. My aim in this paper is to advance a position 
according to which logic is even weaker than the standard two subclassical accounts, that is, weaker than each of K3 and LP.

My argument stems from two fronts. The first front is an account of the role of logic. Asking after the role of logic is useful for understanding the sense in which puzzling phenomena motivate subclassical logic in the first place. The role of logic, on the picture I advocate, is to serve as the (unique) non-empty foundational closure operator for all of our true theories. It is on this front - the role of logic - that monism about logic is correct. ${ }^{1}$ The second front of my argument is the duality of the two target logics and their corresponding philosophical accounts. Such duality is well-known $[7,32,42,50,53]$ but its philosophical consequences are under-appreciated.

The structure of the paper is as follows. $\S 3$ and its subsections sketch the target account of logic and its role. $\S 4$ makes plain the sense in which logic, as understood in $\S 3$, needs to be weaker than classical logic in the face of familiar strange phenomena (e.g., vagueness, antinomies, etc.). With background in place $\S 5$ turns to the main - and very simple - argument from the standard subclassical-logic candidates (viz., K3 and LP) to a weaker subclassical logic. $\S 6$, by considering objections and replies, further illuminates the position, including the sense in which, as the title claims, our best account of logic is one according to which there is no logical negation - at least nothing logically interesting by that name (beyond De Morgan interaction).

\section{The role of logic: universal closure}

There's one usage of 'logic', dominant in mathematical circles, whereby anything that looks sufficiently like a well-defined formal entailment relation (with some sufficiently clear notion of formal in play) is a logic. The pluralism of Beall and Restall [12] is in this family though it imposes conditions on which, among the very many, entailment relations count as logic. I have no interest in disputing such a usage or getting involved in terminological debate.

But there is a philosophical issue. Debates about whether logic is nonclassical are not terminological. If we're to take a stand on whether various phenomena demand nonclassical logic then we need to give some account of logic itself. The target positions and debates do take a stand on whether

\footnotetext{
${ }^{1}$ Pluralism about logic along the lines of Beall and Restall [12] may be compatible with the monism I shall assume, but this deserves further debate (elsewhere). The compatibility, if it is there, may arise from Beall-Restall pluralism being a pluralism about mostly extralogical entailment relations; but only one such relation is logic on the account I give here.
} 
various phenomena demand nonclassical logic - subclassical-logic theorists saying that various phenomena motivate nonclassical (in particular, subclassical) logic. I assume in this paper that such positions are right: logic itself is subclassical (and hence nonclassical) and familiar puzzling phenomena have led us to this position.

A pressing philosophical question arises: what role does logic play that requires logic itself to be weaker than classical logic in the face of puzzling phenomena? And what, if anything, is this special relation called 'logic' that philosophers have long highlighted as marking the strictest of theoretical boundaries - the one boundary beyond which no true theory can transgress.

I suggest that answers to these questions are most naturally found on a familiar account of logic: namely, that logic is the formal entailment relation that serves as the foundational, universal, topic-neutral closure relation for our true theories. Let me take these ingredients in parts, and then turn to the question of how this account lends natural answers to the noted philosophical questions about logic.

\subsection{Logical, topic-neutral vocabulary}

Logical vocabulary is said to be topic-neutral in the sense that it is neutral with respect to the phenomena about which one might theorize; it is vocabulary that may be - and is - safely used in any true theory whatsoever.

What is this vocabulary? The debate rages on; and I have little to add that will push the debate forward. For present purposes I take a fairly traditional and largely uncontroversial approach. The logical vocabulary is standard first-order vocabulary without identity. By 'standard' here I do not mean that the vocabulary obeys standard (viz., classical) logic in all respects. Rather, I mean two things: namely, that there are various expressions of the standard arities and grammatical categories (e.g., binary conjunction, etc.) and that the standard truth-in-a-model conditions for such connectives are largely correct.

While the logical vocabulary on this view is the standard first-order vocabulary - with the only logical conditional (the material conditional) defined via logical negation and logical disjunction - I focus for present purposes only on sentential connectives, namely, the standard quartet: ${ }^{2}$

\footnotetext{
${ }^{2}$ The only logical conditional in this picture is the material conditional (i.e., 'the hook' or 'horseshoe') $A \supset B$, defined per usual as $\neg A \vee B$, where $\neg$ is logical negation (see below). Truth-/falsity-in-a-model conditions for the conditional provided by logic are left implicit below.

In the full first-order picture we have two quantifiers corresponding to logical conjunction
} 
- Unary: logical null operator: it is true that...

- Unary: logical negation operator: it is false that....

- Binary: logical conjunction: ...and....

- Binary: logical disjunction: ...or...(or both).

Note that, on this account, logic has connectives that speak (so to speak) about truth and falsity; however, it speaks only of the topic-neutral truth and falsity operators - not truth or falsity predicates, which go beyond logic. As Ramsey correctly noted [41] the truth operator of which logic speaks is absolutely redundant, and as such most presentations of the stock of logical connectives omits an explicitly recorded truth operator - leaving it to be the invisible null operator [1]. I shall largely follow suit, though it's worth remembering that the basic stock of logical (propositional) connectives is a quartet that falls into two pairs of dual operators.

Parenthetical remark. A truth (dually, falsity) predicate, as deflationists have long correctly noted $[6,21,25]$, is a different matter; it is much like a logical device in its 'nature' but is far from redundant. And while a truth (similarly, falsity) predicate is not among the logical vocabulary it is responsible for creating some of the puzzling phenomena (e.g., truth-theoretic paradoxes) in our broader language that motivate target subclassical-logic theories - more on which below. End remark.

\subsection{Foundational, universal closure relation}

Theories are sets of sentences (or of propositions or etc.). When we form a theory of some phenomenon we throw a bunch of sentences into the theory, namely, all of those sentences that we think are true about the phenomenon. In turn, we require a theory that reflects not just our thrown-in truths; we require a theory that reflects all of the true consequences of the theory's claims. And this is the job for a closure relation: a relation that 'completes' the set of truths by adding all sentences that are consequences of the theory according to the relation.

Logic - that is, the logical consequence relation or logical entailment relation - is one such closure relation. If you close your theory $T$ under

(universal quantifier) and logical disjunction (existential quantifier). Truth-/falsity-in-amodel conditions for predicates (open sentences) are given in terms of extensions/antiextension pairs, and imposing constraints corresponding to 'exhaustion' and 'exclusion' discussed in $§ 3.3$; but I leave details to cited work, since the main issues of this paper may be seen at the propositional level.

Australasian Journal of Logic (14:1) 2017, Article no. 1 
logic then you'll get a theory $T^{\prime}$ which is a superset of $T$ that contains every sentence which is a logical consequence of anything in $T^{\prime}$. Of course, logic tells you only what follows in virtue of the logical vocabulary. Such vocabulary serves as the universal - or topic-neutral - base vocabulary for all of our theories; but consequences tied only to such vocabulary (as in logical consequence) are not enough consequences to serve robust, topic-specific theoretical aims.

Since it looks only at the logical vocabulary logic is not enough to give you all of the true consequences of (for example) our best theory of knowledge. Indeed, logic won't give you any consequences of (for example) knowledge attributions except for the consequences that it gives for any claim whatsoever - namely, the logical consequences. Hence, to complete our theory of knowledge beyond our thrown-in truths about knowledge we must build a closure operator on top of logic, one that specifically looks at the topic of knowledge itself (or minds, or gravity, or numbers, or love, or whatever the phenomenon might be). In the case of a theory of knowledge, one requires as a consequence of (for example) Agnes knows that Max is sleeping more than its mere logical consequences; one requires (for example) that Max is sleeping be in the closed theory too. Such topic-specific consequences will never be achieved by topic-neutral logic. That's why theoreticians work to build extra-logical closure operators on top of logic.

In our true theories the closure operators never transgress logic; but they always go well beyond it - at least in all of the interesting such theories. As a result, the weaker logic itself is, the harder theoreticians must work to construct appropriate closure relations.

On my view logic is even weaker than leading subclassical-logic views take it to be. Rebuilding our proverbial ship at sea [40] is as hard as it is because logic does sorely little to hold things together.

\subsection{Logic itself: entailment via models}

Logical entailment is a necessary truth-preservation relation - or absence-ofcounterexample relation - over possibilities. To make this precise, models are used in the familiar Tarskian way though in subclassical accounts the models differ a bit in affording more 'semantic values' (or semantic categories, etc.).

The logical vocabulary have familiar truth-in-a-model and falsity-in-amodel conditions. A very intuitive way to formally model this is from Dunn $[18,19],{ }^{3}$ where we think of our set of semantic values as the powerset of the

\footnotetext{
${ }^{3}$ And see also Makinson's work [29, pp. 36-7] and Dunn's PhD thesis [18].
} 
standard classical set of two values: $\wp(\{1,0\})$. Intuitively, we think of logic as preserving truth (modeled by value 1 ) as far as logical vocabulary goes, and a sentence's being at least true as modeled by the sentence's having (at least) 1 as a semantic value.

We think of $v(A)$ as the semantic value of $A$, and $1 \in v(A)$ and $0 \in v(A)$, respectively, as $A$ 's being at least true (false) according to $v$. The clauses then are familiar where (for duality's sake) $\dagger$ is the unary it is true that operator and $\neg$ the it is false that operator:

- $1 \in v(\dagger A)$ iff $1 \in v(A)$.

- $0 \in v(\dagger A)$ iff $0 \in v(A)$.

- $1 \in v(\neg A)$ iff $0 \in v(A)$.

- $0 \in v(\neg A)$ iff $1 \in v(A)$.

- $1 \in v(A \wedge B)$ iff $1 \in v(A)$ and $1 \in v(B)$.

- $0 \in v(A \wedge B)$ iff $0 \in v(A)$ or $0 \in v(B)$.

- $1 \in v(A \vee B)$ iff $1 \in v(A)$ or $1 \in v(B)$.

- $0 \in v(A \vee B)$ iff $0 \in v(A)$ and $0 \in v(B)$.

In turn logical consequence is defined to be absence of counterexample in virtue of logical form (where logical form is defined in terms of the logical vocabulary). Specifically, a counterexample to the argument from premises $A_{1}, \ldots, A_{n}$ to conclusion $B$ is a model in which each premise $A_{i}$ contains 1 but $B$ doesn't. An argument $A_{1}, \ldots, A_{n} \therefore B$ is logically valid iff it has no counterexample as far as logic is concerned.

To get the classical account of logical consequence the conditions of exhaustiveness and exclusion are imposed on the truth and falsity relations:

- exhaustion: every model puts either 1 or 0 into the semantic value of every atomic (and, in turn, every sentence);

- exclusion: no model puts both 1 and 0 into the semantic value of any atomic (or, in turn, any sentence).

Adding exactly one of these dual conditions is the standard route of target subclassical logics, to which we now turn. ${ }^{4}$

\footnotetext{
${ }^{4}$ If one wishes to be explicit about the resulting closure operator one may follow Tarski
} 


\section{Strange phenomena and logic}

Strange phenomena - particularly paradoxes - have motivated a dive beneath the water mark of classical logic. The two target directions of the dive are 'paracomplete' and 'paraconsistent'. The question is: how is it that the strange phenomena motivate the dive beneath classical-logic levels? The answer adverts to the role of logic.

\subsection{Paracomplete}

Some phenomena, perhaps such as vagueness, seem to suggest that the complete true theory of the given phenomenon is incomplete with respect to truth-/falsity-operator claims. But if the true theories of any phenomena must respect logical-disjunction behavior (as they must, on the current picture) such phenomena motivate true theories that are both closed under logic and yet free of some excluded-middle claims. But that just cannot happen if logic demands excluded middle. Hence, such phenomena motivate a weaker-than-classical logic in which excluded middle fails. ${ }^{5}$

A leading candidate among subclassical logics is K3. ${ }^{6}$ On this picture logic makes a claim about exclusiveness of truth and falsity but makes no claim about exhaustiveness. No matter the sentence $A$, logic recognizes no

[16] and define logic to be the closure operator induced by the entailment relation above: the logical closure of theory $T$ is the theory $T^{\prime}$ that both includes $T$ and contains all and only the logical consequences of $T^{\prime}$. The threefold properties of closure operators are demanded on this standard account, namely, that where $L(T)$ is the Logical closure of $T$, we have:

- $T \subseteq L(T)$

- $L(T)=L(L(T))$

- If $T \subseteq T^{\prime}$ then $L(T) \subseteq L\left(T^{\prime}\right)$.

These are assumed in my discussion, and are satisfied by all of the target subclassical logics (viz., K3 and LP). For technical discussion related to Tarski's own approach to consequence operators see McGee [31], Sher [47, 48] and, directly related to zeroing down to first-order vocabulary, Feferman [20].

${ }^{5}$ The requisite disjunction behavior is technically called primeness in logic: a theory is prime iff it contains a disjunction just when it contains one of the given disjuncts. This feature of true theories that respect disjunction behavior is motivated by the familiar truthin-a-model conditions for disjunctions. Complaints against supervaluational approaches to disjunctions $[27,49]$ are precisely complaints against non-prime true theories of the relevant phenomena. The subclassical-logic approaches at issue in this paper demand primeness of theories, at least with respect to logical disjunctions.

${ }^{6}$ This is not the only candidate; but my focus is in part on advancing a picture in which if we're to go subclassical we should go below the leading candidates K3 and LP.

Australasian Journal of Logic (14:1) 2017, Article no. 1 
possibility in which both it is true that $A$ and it is false that $A$ obtain. Since there's no such possibility recognized by logic, any argument from an arbitrary contradiction $\dagger A \wedge \neg A$ to an arbitrary sentence $B$ is logically valid - 'explosion' is imposed by logic. But on this picture, logic recognizes many possibilities in which the dual of exclusion (viz., non-exhaustiveness) occurs: as far as logic itself is concerned (i.e., as far as logic's vocabulary and constraints go) there are possibilities in which neither it is true that $A$ nor it is false that $A$ obtain. Accordingly, the logic is 'paracomplete' in the sense that excluded middle (exhaustiveness of logical negation) is not logically valid - not logically imposed.

Parenthetical remark. To get K3 from the truth-in-a-model and falsityin-a-model conditions in $\S 3.3$ one requires that no atomic is related to both value 1 and value 0 . In other words one drops the exhaustion condition and only the exhaustion condition - on models. End remark.

\subsection{Paraconsistent}

The same story goes for glut-theoretic accounts instead of gap-theoretic accounts. Some phenomena, perhaps such as truth-theoretic or propertytheoretic paradox, seem to suggest that the complete true theory of the given phenomenon is inconsistent with respect to truth-/falsity-operator claims. But if logic, qua universal closure operator, demands that the closure of any logically inconsistent theory is the trivial theory (i.e., the set of all sentences of the theory's language) then the true theory of such phenomena is flat-out absurd. Hence, such phenomena motivate a weaker-than-classical logic in which the dual of exhaustion (viz., exclusion) fails.

A standard subclassical logic for such theories is LP. $^{7}$ On this picture logic makes a claim about exhaustiveness of truth and falsity but makes no claim about exclusiveness. No matter the sentence $A$, logic recognizes no possibility in which neither it is true that $A$ nor it is false that $A$ obtains. Since there's no such possibility recognized by logic, any argument from an arbitrary sentence $B$ to an arbitrary 'dual contradiction' $\dagger A \vee \neg A$ is logically valid - excluded middle is imposed by logic. But on this picture logic recognizes many possibilities in which the dual (non-exclusiveness) occurs: as far as logic itself is concerned (i.e., as far as logic's vocabulary and constraints go) there are possibilities in which both it is true that $A$ and it is false that $A$ obtain. Accordingly, the logic is 'paraconsistent' in the sense

\footnotetext{
${ }^{7}$ Again, this is not the only candidate; but my focus is in part on advancing a picture in which if we're to go subclassical we should go below the leading candidates LP and K3.
} 
that explosion (or exclusiveness of logical negation) is not logically valid not logically imposed.

Parenthetical remark. To get LP from the truth-in-a-model and falsityin-a-model conditions in $\S 3.3$ one requires that every atomic is related to either value 1 or value 0 . In other words one drops the exclusion condition and only the exclusion condition - on models. End remark.

\subsection{Summary: closing our true theories}

I have presented what I take to be a familiar account of logic and its role. In light of the given account it is plain to see why strange phenomena (e.g., paradoxes) motivate subclassical logic: various such phenomena motivate glutty theories and various phenomena motivate gappy theories; and the closure role of logic cannot accommodate such theories without being subclassical.

My aim in this paper is not to argue that strange phenomena demand subclassical logic. My aim may be seen conditionally: that if, as I and many others have argued $[3,4,6,11,14,21,24,33,37,51,52]$, the best lesson to draw from various strange phenomena is that logic is subclassical then logic is best seen as being weaker than the leading candidates K3 and LP. It is the consequent of this claim to which I now turn.

\section{From subclassical to really subclassical}

I agree with LP/K3 theorists: we should see logic as subclassical. But, as I did in my long-ago original thinking, I think that we should reject each of the LP and K3 pictures as pictures of logic.

\subsection{One: Dual problems, dual virtues}

One reason to question an LP-vs-K3 or K3-vs-LP stance arises from a wellknown 'duality' (which extends to the philosophical applications). In particular, both LP/K3 have dual problems and dual virtues. Each has an account of logical negation that seems to require the creation of extra-logical resources. Whereas LP glut theorists have no detachable conditional in the vocabulary of logic but do have an identity-satisfying logical conditional (i.e., a conditional $\rightarrow$ such that all sentences of the form $A \rightarrow A$ are true by logic's demands alone), the K3 gap theorist is in the dual position - she enjoys a

detachable conditional in the vocabulary of logic but no identity-satisfying

Australasian Journal of Logic (14:1) 2017, Article no. 1 
logical conditional. ${ }^{8}$ Both camps spend much of their efforts on navigating around the one 'logical failure' or the other by either creating extra-logical resources or invoking extra-linguistic resources altogether (e.g., notions of rejection, acceptance, and so on). In the end it is very difficult to select between them. Witnesses to such dual problems and dual virtues may be found in recent works of target theorists [6, 21, 38, 39].

One widely discussed issue serves as an example: namely, the 'characterization' problem. Consider the strange phenomenon of the liar, where one has a sentence $L$ that says of itself that it is untrue. The LP (glut) theorist has not even a prima facie problem characterizing the status of such a sentence: her theory contains the claim $\dagger L \wedge \neg L$, the claim that $L$ is a glut. And there's no problem with this sentence being in her closed theory, since the theory allows for such sentences without 'exploding' into the trivial theory. On the other hand, there is a prima facie problem with the characterization of normal or non-strange sentences. The natural thought is that normal sentences are 'just true' (if true) or 'just false', and not both. But in LP, as many have noted $[6,46]$, logic itself offers no help here. After all, in LP every sentence of the form $\neg(\dagger A \wedge \neg A)$ is true, and so for any true $A$ every sentence of the form $\dagger A \wedge \neg(\dagger A \wedge \neg A)$ is true, which is a natural expression of 'just true'. Accordingly, the LP theorist needs to invoke extra-logical resources or extra-linguistic resources to perform the task of truly characterizing 'normal sentences' - or argue that such characterization needn't be performed at all.

I invoke the characterization problem not to argue that it is a major problem for LP-based glut theorists. I raise it to illustrate the sort of duality of LP-based versus K3-based positions. The K3-based theorist faces exactly the dual problem. In her case there's no problem utilizing merely logical resources to characterize 'normal' sentences; she simply puts exactly one of $\dagger A$ and $\neg A$ into her closed theory - and that's that. (Logic demands that the theory be absurd - the trivial theory - should both such sentences be in a closed theory.) The problem for the K3 theorist concerns the strange sentences such as L. In twisted sentences such as a liar-like sentence L the K3 theorist cannot use merely logical resources to have a (closed) theory according to which $\mathrm{L}$ is untrue; for such resources only allow the theorist a claim of the form $\neg L$ or (as in 'neither true nor false') $\neg \dagger A \wedge \neg \neg A$. But adding these sentences to a theory closed under K3 results in the trivial theory. Accordingly, the K3 theorist needs to invoke extra-logical resources or extra-linguistic resources to perform the task of truly characterizing 'normal

\footnotetext{
${ }^{8}$ By a detachable conditional I mean a conditional $\rightarrow$ such that $A$ (extensionally) conjoined with $A \rightarrow B$ entails $B$.
}

Australasian Journal of Logic (14:1) 2017, Article no. 1 
sentences' - or argue that such characterization needn't be performed at all.

Problems such as the characterization problem are well-known by truth theorists. Responses to such problems are wide-ranging and varied. My point, again, is not to push such problems as major problems; the point is only to highlight the duality. In general, whatever solution works for the one side on standard problems (e.g., 'just true' or 'neither true nor false' or etc.) has a corresponding solution on the other; and - note well - logic itself is generally doing very little in the solution(s). Both sides wind up either invoking extra-logical resources in the end or explaining away the appearance of would-be logical connectives. These strategies - invoking extra-logical resources or explaining-away responses to problems - are important in the end; but they don't tilt towards LP over K3 as logic or K3 over LP as logic.

\subsection{Two: Theoretically ugly lopsidedness}

A bigger reason to reject each of the LP and K3 pictures of logic is that each seems to be unnaturally 'lopsided' or biased with respect to truth and falsity operators: the $\mathrm{K} 3$ picture is one in which logic precludes having both it is true that $A$ and it is false that $A$ jointly obtain for some $A$, but on this K3 picture logic does not preclude the perfect dual, namely, having neither it is true that $A$ nor it is false that $A$ obtain for some $A$. And the LP picture is equally lopsided with respect to the truth and falsity operators: here, logic

recognizes possibilities in which both $\dagger A$ and $\neg A$ obtain, but logic precludes any 'gap' - precludes having neither $\dagger A$ nor $\neg A$ obtain.

This is imbalanced in a bad way. And when we are trying to give a natural picture of logic such lopsidedness is glaringly ugly. Why would logic make a claim only about one of exclusion and exhaustion [6, Ch. 4]? A classical theorist rightly finds such an unmotivated lopsidedness to be objectionable; and she may reject the dive to either LP or K3 for that reason. For those of us who are committed to the dive beneath the classical level we have to find a deeper source of balance. Fortunately, there are well-known destinations that fit the bill.

\subsection{Towards FDE: more natural}

Given the duality issue and the lopsidedness issue, we should - I suggest settle on at least FDE, the so-called 'first-degree entailment logic' or 'logic of tautological entailments' $[1,19]$. And the reasons for adopting at least the FDE picture over the more common LP or K3 are as follows.

Australasian Journal of Logic (14:1) 2017, Article no. 1 


\subsubsection{Paradoxes still naturally resolved}

Logic, on this 'deeper' picture, still affords a natural treatment of the paradoxes. The 'solutions' afforded by standard (though lopsided) subclassical logics carry over to FDE. The logic is weak (or 'deep') enough to accommodate standard paradoxical notions (e.g., truth, exemplification, etc.) [50, 53]. By diving deeper than the standard lopsided subclassical levels we do not lose the options for naturally resolving paradoxes; we have more options treating some of them as 'gappy phenomena' and some 'glutty phenomena' versus trying to squeeze them all into one category or the other, regardless of how unnatural the fit appears.

\subsubsection{Goodness is preserved}

Any goodness enjoyed by the logic-is-LP or logic-is-K3 pictures is preserved in the FDE picture. Moreover, the usual extra-logical resources invoked to carry out work that, according to standard subclassical solutions, was erroneously attributed to logic itself (e.g., acceptance-rejection behavior, or additional theory-specific vocabulary or connectives, etc.) is seamlessly preserved in the FDE picture. Indeed, since classical logic properly extends FDE (as it does LP and K3), all of the classical-logic-based resources are available to the FDE-based theorist, just as they are available to the lopsided K3-/LP-based theorists. (For more on this topic see $\S 6$.)

For now, the important point is that by diving deeper than the imbalanced K3 and LP pictures of logic we do not lose any of the benefits that such approaches enjoy; the only difference is that some of the logical resources that they individually enjoy (e.g., a detachable logical conditional, as in K3, or an identity-satisfying logical conditional, as in LP) are gone; we have such resources, if at all, only by way of extra-logical resources - a topic discussed further in $\S 6$.

\subsubsection{Some striking badness removed}

Preserving goodness is one thing; removing the badness is another. Importantly, not only do we preserve whatever goodness is had by the K3/LP pictures of logic but by adopting a logic-as-FDE picture we avoid the unnatural and inelegant imbalance of the old LP-/K3-based pictures.

If we cared not at all about having a natural and balanced view of logic in our philosophy of puzzling phenomena then removing such badness wouldn't be important. But even the most pragmatic philosophers among us should 
care about theoretical elegance and naturalness; and the FDE-based picture is plainly more elegant and balanced than the standard subclassical leaders.

One might agree that while the badness of a lopsided picture of logic is indeed removed the other badness - involving 'characterization' as in $\S 5.1-$ is doubly bad: the problems that LP-based theorists had are now doubled by taking on the K3-based problems. But this misses the point noted in $\S 5.3 .2$ : those problems are already resolved by invoking extra-logical (often extra-linguistic) resources; and those solutions are seamlessly preserved in the simpler, more balanced FDE picture of logic. ${ }^{9}$

\section{Objections and Replies}

I have given a very simple argument for seeing FDE, as against LP/K3, as logic proper. Against the simple - and, by my lights, forceful - considerations towards FDE there are a variety of objections - including one to which the paper's title is directed. Towards further sketching the target position this section voices and answers the most pressing objections. Where $i$ is a positive integer objections and responses are marked as $O i$ and $R i$ respectively.

O1. Logical negation? From a classical perspective one now wonders what's left of logical negation - of the falsity operator. On your picture, not enough! On your picture logic says virtually nothing about the falsity operator on its own, but at most comments on its interaction with the connectives that logic does characterize on their own. One way to see this is via the standard sequent conditions on logical negation:

$$
\neg \mathrm{L}: \quad \frac{\Gamma \vdash A, \Delta}{\Gamma, \neg A \vdash \Delta} \quad \neg \mathrm{R}: \quad \frac{\Gamma, A \vdash \Delta}{\Gamma \vdash \neg A, \Delta}
$$

Figure 1: Standard falsity-operator (logical negation) rules

These rules reflect the standard thought that logic makes a claim of flipflop behavior with respect to the truth and falsity operators - or, in short, on would-be logical negation. (And with these conditions come the

\footnotetext{
${ }^{9} \mathrm{As}$ in $\S 5.1 \mathrm{I}$ am not advancing the characterization problems as knockdown problems; they serve rather to highlight the duality of the philosophical positions grounded in an LP-based versus K3-based picture of logic itself. As noted in $\S 5.1$ some theorists often try to explain away the prima facie characterization problems that confront the given positions. Inasmuch as those explain-away strategies work (if at all) they work for the FDE-based picture of logic too.
}

Australasian Journal of Logic (14:1) 2017, Article no. 1 
exclusive behavior reflected in $\mathrm{K} 3$ and the exhaustive behavior reflected in LP.) But such conditions are simply absent in FDE. On your picture, logic makes no such claims about a would-be 'logical negation' - and so makes no claims about stand-alone negation behavior. All that logic says about a would-be 'logical negation' is what it says about the falsity operator's interaction with other logical connectives (e.g., $\neg \vee, \neg \wedge$ or etc.). There's nothing it says about logical negation on its own (so to speak).

Moreover, this objection is not restricted to a classical-logic complaint. A similar complaint comes from the LP and K3 camps themselves. While neither LP nor K3 has the standard classical sequent negation rules, each picture does have logic declaring something about negation's (logical) behavior on its own. On the LP picture (or $\mathrm{LP}^{+}$picture, which is LP generalized to a multiple-conclusion relation) we have logic declaring the exhaustive behavior of negation:

$$
B \vdash_{L P+} A, \neg A
$$

while the K3 picture declares the dual:

$$
A, \neg A \vdash_{K 3+} B
$$

On the FDE picture of logic there simply is no stand-alone negation behavior that logic itself describes.

In summary: instead of giving a more natural picture of logic you've gotten rid of logical negation altogether!

R1. Correct. That there is no logical negation - or no interesting such connective - might be seen as too radical to be plausible. I do not share this view at all. In fact, that logic imposes no such constraints on the falsity operator is the much more natural view than the standard subclassical views. The opposing (subclassical) views, as already said, carry an unnatural inelegance. On those views logic demands that the falsity operator be exhaustive only, or that it be exclusive only. Saying this, I admit, only repeats the argument from LP/K3 to FDE in $\S 5$; but that we're left with no interesting logical negation is a powerful objection to the view only if the most (or even a more) natural picture of logic needs to involve such a connective, a connective which logic characterizes in stand-alone terms, so to speak. And I don't see that that's so. There is a logical connective called negation or falsity operator; it's just that logic imposes no interesting constraints on it (or, dually, the null or truth operator), aside from what logic demands of its interaction with other logical connectives.

Australasian Journal of Logic (14:1) 2017, Article no. 1 
O2. Negation and itself. In R1 you endorsed the claim that logic makes no claims about 'logical negation' except concerning its behavior with other (logical) connectives - connectives about which logic does impose constraints. But that's not right if 'other connectives' requires distinct other connectives; after all, the FDE picture of logic involves the constraint of double-negation equivalence - and so, on that picture, logic makes a serious claim about negation's interaction with itself.

R2. Correct. If logic really is FDE and not something weaker, then 'other connectives' doesn't require distinctness. Objection $\mathrm{O} 2$ is right on that score.

O3. Towards Ockham? Granted: if we adopt a subclassical-logic picture we should go deeper than LP and K3 to something at least as deep as FDE, for reasons you've given. But there's something prima facie strange about FDE's demand of double-negation equivalence on top of an otherwise pure and simple De Morgan picture of logic. Indeed, considerations of simplicity and naturalness suggest diving deeper to the depth of no more nor less than pure De Morgan constraints - that logic itself imposes no more than De Morgan interaction with respect to logical vocabulary. On this picture double-negation equivalence should be gone; and all that we have left of 'logical negation' are traces in the form of 'negative operators' (such as $\neg \vee$ or $\neg \wedge$ or the like); and these, together with the stand-alone connectives that logic does describe (such as logical disjunction and logical conjunction), are what secure the deep (weak) essence of logic - the De Morgan patterns. Your arguments drive deeper than FDE to what Urquhart calls 'Ockham logic' (which characterizes so-called Ockham algebras, following work of Berman [13]). This is the pure De Morgan picture in which one drops the prima facie ad hoc demand of double-negation equivalence. ${ }^{10}$

R3. My chief concern is to dive at least as deep as FDE for reasons given. Objection O3 grants as much but carries the debate towards a deeper dive. ${ }^{11}$ While I'm very sympathetic with the Ockham picture I think that

\footnotetext{
${ }^{10}$ For those familiar with the Routley-Routley Star approach to negation: as is clear from Urquhart's discussion Ockham logic is the logic one gets from imposing no conditions on the star operator (and, a fortiori, dropping the usual condition that the star mate of the star mate of a world is that world). Another way of thinking about Ockham is that one gets it by dropping the requirement of double-negation equivalence. That one needs to impose the double-star or double-negation conditions in order to achieve double-negation equivalence may suggest an air of ad hocery - and if so, one may be led to Ockham as the more natural picture than FDE. But I leave this for future debate.

${ }^{11}$ And lest one push for a slippery slope to the null logic, let me simply say that I find the option to be interesting but, at least without further argument, too far down the
} 
there are considerations in favor of double-negation equivalence (and so against Ockham) from a 'pure De Morgan' perspective.

I agree that, on the picture I'm advancing, the salient heart of logic amounts to little more nor less than De Morgan patterns of logic's connectives. But while logic says nothing interesting about stand-alone falsity-operator behavior or stand-alone truth-operator behavior it does say something about De Morgan patterns of such operators working together, including familiar examples such as

$$
\neg(\neg A \vee \neg B)-\vdash \dagger A \wedge \dagger B
$$

and

$$
\neg(\neg A \wedge \neg B) \dashv \vdash \dagger \vee \dagger B
$$

But not only does logic say something about its dual conjunctions/disjunctions (similarly, in the fuller story, universal/existential quantifiers) working with truth and falsity operators, it says something in the elementary case of truth and falsity operators interacting together:

$$
\dagger \neg A \dashv \neg \nmid A
$$

and in turn

$$
\neg \neg A \dashv \vdash A \dashv \vdash \dagger \uparrow
$$

So, inasmuch as patterns of De Morgan behavior over its connectives (with dual operators transforming into dual operators) form the beginning and end of logic's constraints, there is a clear sense in which double-negation equivalence is an essential part of the picture. ${ }^{12}$

I do find the simple Ockham picture (which is basically FDE sans double-negation equivalence) to be a plausible account of logic; and it is in keeping with the general thrust of this paper. But I leave the debate over Ockham versus FDE for future debate.

O4. What of Symmetric (S3)? If you want to restore symmetry and remove lopsidedness from K3/LP, why not go for the strongest logic that allows you to do so? After all, the intersection of K3 and LP is a natural approach towards balance. FDE is not the intersection of K3 and LP; the

rabbit hole to afford a natural, plausible picture of logic.

${ }^{12}$ Correspondence with Francesco Paoli helped to clarify my thinking on this point. Paoli's work [34] independently comes to similar conclusions.

Australasian Journal of Logic (14:1) 2017, Article no. 1 
intersection is the logic S3. ${ }^{13} \mathrm{~S} 3$ is a reasonably symmetric and aesthetically pleasing picture of logic that would seem to be motivated by your arguments. Your arguments push more for S3 than FDE.

R4. I agree that S3 is a better picture of logic than the leading subclassical ones, for reasons given. Moreover, if the only problem with LP and K3 is their respective lopsidedness then a highly natural resolution towards balance is to take their intersections, landing us in S3 as the objection rightly claims. But on the picture of logic I'm advancing logic demands neither exclusion nor exhaustion of truth and falsity operators, and hence does not impose the residue of these restrictions that is found in the intersection of LP and K3, including, for example, the key S3 principle:

$$
\dagger A \wedge \neg A \vdash \neg B \vee \dagger B
$$

An arbitrary contradiction's logically implying an arbitrary dual contradiction is unmotivated - unless one already thought that logic imposes residue of exhaustion and exclusion, which is what S3 reflects. But on the picture I'm advancing logic demands no trace of exclusion or exhaustion; and so the dive below S3 to the more natural FDE is motivated.

O5. What of classical negation? You say that there is no logical negation, at least nothing that logic itself characterizes apart from interaction with other stand-alone connectives. How, then, do you explain the apparent indeed, screamingly apparent - ubiquity of classical negation?

R5. If it looks like classical negation, it isn't logical negation. Instead, it is something else - some theory-specific negation that has its behavior demanded by the phenomenon in question, not demanded by logic. The behavior is imposed not by logic itself but rather imposed by our various theory-specific closure relations.

\footnotetext{
${ }^{13}$ The name 'S3' is from Field [21] for 'symmetric 3-valued' but the logic itself has been explored at least since the Pittsburgh relevance program [2]. Here, one has three options for sentences (viz., true, 'strange' - my word - and false, linearly ordered just so with 'strange' being higher than falsity but lower than truth); and one defines logical consequence as absence of counterexample, where a counterexample to $A_{1}, \ldots, A_{n} \therefore B$ is a model in which $B$ has a value strictly lower than the lowest $A_{i}$ value. Proof-theoretically one simply adds the following rule to a complete proof system for FDE to get S3:
}

$$
\dagger A \wedge \neg A \vdash \neg B \vee \dagger B
$$

where $\dagger$ is our (redundant) truth operator. This rule, while valid in both LP and K3, is invalid in FDE. (A counterexample in FDE involves glutty $A$ and gappy $B$.) 
Background example. Recall that logic has nothing special to say about (for example) the knowledge operator; logic says about knowledge claims what it says about kitten claims, quantum-mechanical claims, theological claims, and so on - it doesn't say anything about such claims except what logic imposes on their interaction with logical vocabulary. It's not logic that tells us that it is known that $P$ delivers $P$. The delivery of $P$ from it is known that $P$ is pulled off via a closure relation built on top of logic. And that's what we do as theorists: we build closure or entailment relations, all of which enjoy logic as the universal (topic-neutral) foundational closure relation.

Returning to the explicit objection: how, then, do we explain the apparent ubiquity of classical-negation behavior in our theories if there is no interesting logical negation - certainly no classical negation involved in logic? The answer is not different in spirit from the case of knowledge or many other bits of extra-logical vocabulary: the appearance of classical-negation-like behavior is imposed by theory-specific closure relations. (The same is true of conditionals and the like.) In particular, apparent classical-negation behavior is achieved within topic-specific theories (e.g., of phenomenon $P$ ) by imposing 'shriek' and 'shrug' rules on the given theory's extra-logical closure relation $\vdash_{T}[8,9]:{ }^{14}$

Exh. $B \vdash_{T} \forall x(P x \vee \neg P x)$.

Exc. $\exists x(P x \wedge \neg P x) \vdash_{T} B$.

We use these rules to narrow the class of (non-trivial) models for our theories, and define our theories' entailment relations (closure relations) over those models. Perhaps most of our substantive theorizing is backed by such rules, and overturned only when the given phenomenon screams out for a different treatment [10].

This picture is not different from the standard thought of, for example, intuitionists who think that while logic doesn't impose certain doublenegation behavior, our best theories of various phenomena impose such behavior (by way of theory-specific entailment or closure rules). ${ }^{15}$

\footnotetext{
${ }^{14}$ The 'shriek' name comes from a not-uncommon practice, possibly initiated in [38], of using ' $p$ ' to abbreviate $p \wedge \neg p$ (though I've pushed the shriek or 'bang' symbol to the front for readability); and the term 'shrug' seemed an appropriate name (suggested by Colin McCullough-Benner) for the dual, and this may be symbolized $i p$. The shriek rules have the form of (Exc) above, while the shrug rules have the form of (Exh). Again, see [8, 9] for details of the applications in mind.

${ }^{15}$ Lloyd Humberstone and A. J. Lock very briefly gesture in the direction of this idea in a paper arising from investigations of logical negation $[26, \S 1$, p. 431]:
}

Australasian Journal of Logic (14:1) 2017, Article no. 1 
O6. Garfield's Dog. Jay Garfield [22] argues that logic is weaker than the standard LP/K3 alternatives, and seems to suggest a logic at least in the vicinity of FDE. How is your position different from Garfield's?

R6. Garfield and I agree that if logic is subclassical then it's deeper than the leading subclassical approaches have it - deeper than K3/LP. But we come at the question from very different philosophical positions on logic. Garfield's given argument(s) rest on an epistemic conception of logic, or at least a very tight tie between epistemology and logic in which logic has a salient prescriptive role in 'reasoning'. On my view, while logic has a foundational role to play in rational inquiry the role is rather more limited than Garfield's arguments require. On my view logic is the unique foundational closure operator on our theories; it is a necessary truthpreserving relation in virtue of logical vocabulary, and is independent of epistemology. Unlike Garfield, I am in large agreement with the Harman picture [23] as adapted in recent subclassical work [10].

O7. Agnosticism? Routley and Meyer, long ago [45], pushed for a picture of logic in the FDE vicinity. But unlike you they argued from similar considerations (e.g., symmetry, duality of problems, etc.) that with FDE as logic we should be agnostic about gluts and, by symmetry, gaps that is, neither accept nor reject that the full truth of the world is glutty (similarly gappy). ${ }^{16}$ Why is such agnosticism misplaced?

R7. The given case for such agnosticism is by my lights dubious; it requires that logic itself be the chief force behind at least paradox-driven gluts or, similarly, gaps. (Such cases were largely the focus of Routley and Meyer; and subsequent philosophers also focus on logic-driven gluts/gaps too.) If that were right - if logic's constraints were required for accepting glutty or dually gappy theories - then the Meyer-Routley agnosticism would be

such a package [such as the FDE picture I am advancing in this paper] need not be motivated by an outright rejection of LEM: it would he sufficient to regard this as a (possibly correct) substantive metaphysical principle not appropriately built into one's account of the logic of the connectives concerned.

Humberstone and Lock do not develop the idea, but what they say (above) is in the spirit of the basic idea that I am currently advancing: namely, that logical negation is neither 'exhaustive' or 'exclusive' (i.e., logic doesn't validate excluded middle or explosion), but many of our best (true) theories impose as much by theory-specific constraints on the theories' respective entailment (closure) relations.

${ }^{16}$ Similar considerations, though not clearly with a push for an FDE-only picture of logic, come from T. Parsons [35, 36], E. Mares [30], V. McGee [32], and G. Restall [42], and maybe even from early theorists such as Martin-Woodruff [53] and Dowden [17].

Australasian Journal of Logic (14:1) 2017, Article no. 1 
appropriate. But I think that there not only can be other motivations for (say) gluts but that there are other such motivations. And this is an important part of the picture that I am advancing. (See next objection.)

O8. But now why gluts/gaps at all? You've weakened logic to the point where not even the standard strange phenomena (particularly paradoxes) motivate gluts - or dually gaps! ${ }^{17}$ For simplicity, concentrate just on gluts. The problem: if logic doesn't impose exhaustion on all of our theories then we lose all motivation for gluts - or at least all paradox-driven motivation. Right? (The same can be said for standard gappy approaches.)

R8. No. Here are two paradox-driven arguments for gluts where we have no excluded middle in play. (I give these not only as examples, but of thumbnail sketches of arguments that I in fact endorse.)

\section{Naturalness!}

(a) Theoretical beauty and simplicity: Liar-like phenomena prima facie look glutty; they cry out for treatment as odd twisted gluts of truth and falsity. (I'm false or neither true nor false. A very natural reply: Yes, you are.) If it weren't for clinging to a picture of logic that outright excludes such an option then this prima facie aesthetically salient option would be a top contender. Theoretical virtues of beauty, simplicity and naturalness push for as much. Unlike in the standard argument logic doesn't force the glut; but logic certainly allows the option - and in turn other theoretical virtues may lead us to recognize occupants of the space (most notably liar-like phenomena).

(b) Balanced Metaphysics: any indeterminacy in the world should be mirrored by corresponding overdeterminacy. It is a metaphysically lopsided picture to have either indeterminacy alone or overdeterminacy alone. If, as standard subclassical (paracomplete) approaches claim, we have reason to embrace some interdeterminacy in the world then, by a principle of metaphysical symmetry, we should expect overdeterminacy too. ${ }^{18}$ Whether the driving principle of metaphysical symmetry or balance de-

\footnotetext{
${ }^{17}$ I note that Garfield's position discussed in R4 does not answer this pressing question. He adverts to the standard argument from liars to gluts as a reason to embrace gluts [22, p. 241]; but that argument relies specifically on excluded middle being enforced by logic.

${ }^{18}$ One might, in this context, invoke the spirit of a Wittgensteinian remark advanced by Priest [37]: only narrow-minded superstitious communities would rule out one side (overdeterminacy/indeterminacy) over the other (indeterminacy/overdeterminacy).
}

Australasian Journal of Logic (14:1) 2017, Article no. 1 
mands equal degrees of indeterminacy and overdeterminacy is for separate debate. For present purposes such a principle need only demand some degree of overdeterminacy in the face of some degree of indeterminacy.

2. Methodological quest for completeness: we are driven not by logic but by a methodological quest to resolve as many sentences into The True and The False as we can. This sort of methodological commitment may drive towards a resolution of paradoxical sentences into gluts. In particular, while logic won't demand that a typical liarlike sentence be treated as a glut the methodological drive towards completeness - treating sentences either as true or as false subject to overall simplicity and theoretical elegance - may push us to treat it as a glut. (In fact, as above, I believe that such considerations do so push us. But my aim in this reply is to refute O6 by showing that there are excluded-middle-independent arguments for gluts. And similar remarks can be given in the dual case of gaps and other puzzling phenomena.)

Obviously, neither of the foregoing paradox-driven arguments towards gluts have the force of austere logic itself backing the gluts; but I think that such arguments are nonetheless viable - and indeed persuasive. ${ }^{19}$

O9. Only as weak as truth demands? If truth is driving us below classical logic, why not go with the strongest logic compatible with truth's demands? ${ }^{20}$

R9. This sort of thinking partially fueled my previous commitment to excluded middle (and in fact LP), but as I have made clear the choice between LP and K3 carries a theoretical ugliness - and indeed may ultimately be unfounded (possibly even arbitrary), as Beall [6, Ch. 4] suggests. And that matters to the philosophical project. If our only goal were

\footnotetext{
${ }^{19}$ I note that Graham Priest [38] has long argued that versions of Berry's paradox deliver gluts while not in any way requiring excluded middle as a logical principle. If he's right, this is a very important fact, and would only help to refute (O6). But I have doubts that Priest is right about this. Very recently, Ross Brady [15] shows clearly what others vaguely suspected: Priest's clearest 'proofs' of gluts from Berry's paradox rely on excluded middle. See also Field's remarks [21]. The dialectic remains tricky and open, but I assume - with the current objection - that, aside from my arguments above, the extant arguments from paradox to gluts involve excluded middle. If this is wrong, it only helps my case for gluts in a more natural, more balanced picture of logic.

${ }^{20}$ See Hartry Field's remarks at the beginning of his Saving Truth from Paradox [21]. This objection was voiced independently by various participants in the UniLog conference in Istanbul.
}

Australasian Journal of Logic (14:1) 2017, Article no. 1 
to 'save truth' regardless of the broader philosophical picture of logic and its relation to strange phenomena, then perhaps the running objection would have force. But, qua philosophers (vs mere logical technicians), we should care about presenting as natural a picture of logic as we can compatible with truth's demands. And that's what I've suggested leads us deeper than the leading stronger-than-FDE picture.

O10. Jumping up rather than diving down. David Ripley [43] joins you in arguing that the accounts of logical negation in LP and K3 are to be rejected. His objections rely more on an account of meaning than on the aesthetic considerations that you advance. While his arguments (from compositionality of meaning) may not extend to the weaker picture of logic that you advance his supplemental position is a challenge to yours. On his position puzzling phenomena don't challenge any of the classically valid forms; they instead challenge certain (let us say) background-formal features (specifically, so-called structural-rule features) of otherwise classical logic. On his position logical negation is classical negation! The paradoxes, on his view, simply show us that the results of repeated applications of logical consequence cannot safely be chained together without limit. What's important to note is that classical logic is as balanced as FDE logic in your sense (e.g., you have no standard negation-by-itself rules and thereby achieve balance, while Ripley has all standard negation-by-itself rules and thereby achieves balance); but Ripley's position enjoys a clear explanation for the apparent ubiquity of classical negation - namely, that there is a logical negation and it's classical negation! In short, Ripley's picture agrees that puzzling phenomena motivate a deviation from the standard account of classical logic; and Ripley's picture agrees that the leading subclassical-logic pictures are imbalanced. But instead of responding to the imbalance by going even weaker - as you do - Ripley shows that we can enjoy the natural balance of classical logic (suitably understood) while nicely dealing with the paradoxical phenomena.

Put differently, Ripley's position [43] agrees that if puzzling phenomena motivate subclassical logic then, given at least considerations of naturalness and balance, such phenomena motivate a deeper-than-standard subclassical logic (i.e., deeper than LP or K3). But considerations of naturalness and balance do not motivate a rejection of classical logic. And that's where Ripley's position is superior to yours: we should reject that a deeper-than-standard subclassical logic is the best picture of logic since we should reject the need for subclassical logic in the first place.

Australasian Journal of Logic (14:1) 2017, Article no. 1 
R10. I agree that the real choice - at least for transparent-truth theorists $[6$, $21,43]$ and their sympathizers - is between something in the vicinity of Ripley's 'classical logic' and my position involving FDE as logic. While much more debate is needed to decide between these candidates there is at least one reason - possibly two (or even three) - for embracing the subclassical option.

One reason for embracing the subclassical option over Ripley's picture is that, as noted above, some phenomena cry out for a glutty treatment (similarly, other phenomena cry out for gappy treatments). The cries are not fueled by logic; they are fueled by the apparent nature of the phenomena in question. By my lights a sentence that says of itself nothing more nor less than that it's false is - just by the nature of the thing most naturally treated as true and false. Neither the transparent-truth rules nor logic demands as much; and on that Ripley and I agree. But to rule out this natural theory of such phenomena - to rule out glutty theories entirely - strikes me as under-motivated. ${ }^{21}$

Another (possible) reason for rejecting Ripley's account over the account I give concerns the role of logic as universal closure operator on all of our theories - where a closure operator has its standard features (including idempotency or 'unrestricted transitivity'). If one endorses the picture in which logic's chief role is to serve as - or just be - the universal closure operator on top of which all of our theoretical closure operators are built then Ripley's picture may confront problems, due to restrictions on transitivity of logical consequence imposed by Ripley's picture.

\section{Closing remarks}

I've argued that if we have reason to go subclassical then we have good reason to go deeper than the standard LP/K3 frameworks to something at

\footnotetext{
${ }^{21}$ Another possible reason for embracing the subclassical option over Ripley's is that it may afford a more natural account of how we express various important notions in our theories - though, let me emphasize, this is a matter for further debate. (I put it in a footnote because I am least confident in this consideration.) In particular, some of the important theoretical vocabulary involved in Ripley's philosophical position - including various notions of assertion and denial and the like - are not clearly expressible in his target theories due to logical constraints. On its own I do not think that this is a major defect (and, indeed, think that many apparently inexpressible notions are in fact families of limited stratified notions, something that Ripley can easily embrace); but my suspicion is that were his picture of logic itself to be nearer the FDE level then such worries would not arise.
}

Australasian Journal of Logic (14:1) 2017, Article no. 1 
least as deep as FDE. One upshot is that there is no philosophically interesting logical negation (beyond De Morgan patterns); the familiar behavior of so-called logical negation is in fact behavior imposed by individual theories - and in particular their respective closure relations. Such theories impose constraints of exhaustion and exclusion, which are warranted, by the theorist's lights, by the phenomena in question. Such constraints are not imposed by logic itself.

With respect to gluts I've also argued that despite losing the argument from logic to gluts we still have reason to be glut theorists - from both naturalness (treating, e.g., the Liar as it naturally appears) and 'metaphysical balance', if nothing else. But I admit that there's much more debate to be had. My hope is that this paper serves to stimulate such debate. ${ }^{22}$

\section{References}

[1] Alan Ross Anderson and Nuel D. Belnap. Entailment: The Logic of Relevance and Necessity, volume 1. Princeton University Press, Princeton, 1975.

[2] Alan Ross Anderson, Nuel D. Belnap, and J. Michael Dunn. Entailment: The Logic of Relevance and Necessity, volume 2. Princeton University Press, Princeton, 1992.

[3] F. G. Asenjo. A calculus of antinomies. Notre Dame Journal of Formal Logic, 7(1):103-105, 1966.

[4] F. G. Asenjo and J. Tamburino. Logic of antinomies. Notre Dame Journal of Formal Logic, 16(1):17-44, 1975.

[5] Jc Beall. Transparent disquotationalism. In Jc Beall and B. ArmourGarb, editors, Deflationism and Paradox, pages 7-22. Oxford University Press, Oxford, 2005.

[6] Jc Beall. Spandrels of Truth. Oxford University Press, Oxford, 2009.

\footnotetext{
${ }^{22}$ I am grateful to participants at UniLog in Istanbul for lively and helpful discussion of an early draft of this paper. And for comments or discussion I am also very grateful to Ross Brady, Aaron Cotnoir, Mark Colyvan, Diogo Dias, Mike Dunn, Elena Ficara, Jay Garfied, Jared Henderson, Lloyd Humberstone, Dom Hyde, Matthias Jenny, Michael Lynch, David Makinson, Ed Mares, Colin McCullough-Benner, Vann McGee, Julien Murzi, Francesco Paoli, Graham Priest, Greg Restall, Dave Ripley, Marcus Rossberg, Josh Schechter, Kevin Scharp, Lionel Shapiro, Shawn Standefer, Andrew Tedder, and Zach Weber.
}

Australasian Journal of Logic (14:1) 2017, Article no. 1 
[7] Jc Beall. $\mathrm{LP}^{+}, \mathrm{K}^{+}, \mathrm{FDE}^{+}$and their classical collapse. Review of Symbolic Logic, 6(4):742-754, 2013.

[8] Jc Beall. Shrieking against gluts: the solution to the 'just true' problem. Analysis, 73(3):438-445, 2013.

[9] Jc Beall. A simple approach towards recapturing consistent theories in paraconsistent settings. Review of Symbolic Logic, 6(4):755-764, 2013.

[10] Jc Beall. Free of detachment: Logic, rationality, and gluts. Nô̂s, 49(2):410-423, 2015.

[11] Jc Beall and Mark Colyvan. Looking for contradictions. Australasian Journal of Philosophy, 79(4):564-569, 2001.

[12] Jc Beall and Greg Restall. Logical Pluralism. Oxford University Press, Oxford, 2005.

[13] Joel Berman. Distributive lattices with an additional unary operation. Aequationes Mathematicae, 16(1-2):165-171, 1977.

[14] Francesco Berto. Absolute contradiction, dialetheism, and revenge. Review of Symbolic Logic, 7(2):193-207, 2014.

[15] Ross Brady. The use of definitions and their logical representation in paradox derivation. Presented to Universal Logic Congress 5, Istanbul University, Turkey, 2015.

[16] John Corcoran and Alfred Tarski. What are logical notions? History and Philosophy of Logic, 7(2):143-154, 1986.

[17] Bradley Dowden. Accepting inconsistencies from the paradoxes. Journal of Philosophical Logic, 13:125-130, 1984.

[18] J. Michael Dunn. The Algebra of Intensional Logics. PhD thesis, University of Pittsburgh, 1966.

[19] J. Michael Dunn. Intuitive semantics for first-degree entailments and 'coupled trees'. Philosophical Studies, 29:149-168, 1976.

[20] Solomon Feferman. Logic, logics, and logicism. Notre Dame Journal of Formal Logic, 40(1):31-54, 1999.

[21] Hartry Field. Saving Truth from Paradox. Oxford University Press, Oxford, 2008.

Australasian Journal of Logic (14:1) 2017, Article no. 1 
[22] Jay Garfield. 'To Pee and Not to Pee?' Could that be the question? (Further Reflections on The Dog). In Graham Priest, Jc Beall, and B. Armour-Garb, editors, The Law of Non-Contradiction, pages 235244. Oxford University Press, Oxford, 2004.

[23] Gilbert Harman. Change in View: Principles of Reasoning. MIT Press, Cambridge, MA, 1986.

[24] Leon Horsten. The Tarskian Turn: Deflationism and Axiomatic Truth. MIT Press, 2011.

[25] Paul Horwich. Truth. Blackwell, Oxford, 1998. First edition published in 1990.

[26] I. L. Humberstone and A. J. Lock. Semicomplemented lattices and the finite model property. Zeitschrift fur mathematische Logik und Grundlagen der Mathematik, 32(25-30):431-437, 1986.

[27] Rosanna Keefe. Theories of Vagueness. Cambridge University Press, 2000 .

[28] S. C. Kleene. Introduction to Metamathematics. North-Holland, 1952.

[29] David Clement Makinson. Topics in Modern Logic. London: Methuen, 1973. Distributed by Harper \& Row Publishers, Inc., Barnes and Noble Import Division.

[30] Edwin Mares. Relevant Logic: A Philosophical Interpretation. Cambridge University Press, Cambridge, 2004.

[31] Vann McGee. Logical operations. Journal of Philosophical Logic, 25(6):567-580, 1996.

[32] Vann McGee. Ramsey's dialetheism. In Graham Priest, Jc Beall, and Bradley Armour-Garb, editors, The Law of Non-Contradiction. Clarendon Press, 2004.

[33] Chris Mortensen. Inconsistent Mathematics. Kluwer Academic Publishers, 1995.

[34] Francesco Paoli. Bilateralism meets minimalism. Talk delivered at the CLMPS, Helsinki, August 2015, 2015.

[35] Terence Parsons. Assertion, denial, and the liar paradox. Journal of Philosophical Logic, 13:137-152, 1984.

Australasian Journal of Logic (14:1) 2017, Article no. 1 
[36] Terence Parsons. True contradictions. Canadian Journal of Philosophy, 20:335-353, 1990.

[37] Graham Priest. The logic of paradox. Journal of Philosophical Logic, 8:219-241, 1979.

[38] Graham Priest. In Contradiction. Oxford University Press, Oxford, second edition, 2006. First printed by Martinus Nijhoff in 1987.

[39] Graham Priest, Jc Beall, and B. Armour-Garb, editors. The Law of Non-Contradiction. Oxford University Press, Oxford, 2004.

[40] Willard van Orman Quine. Word $\&$ Object. MIT Press, Cambridge, MA, 1960.

[41] F. P. Ramsey. Facts and propositions. Aristotelian Society Supplementary Volume, 7:153-170, 1927.

[42] Greg Restall. Laws of non-contradiction, laws of the excluded middle and logics. In Graham Priest, Jc Beall, and B. Armour-Garb, editors, The Law of Non-Contradiction, pages 73-85. Oxford University Press, Oxford, 2004.

[43] David Ripley. Paradoxes and failures of cut. Australasian Journal of Philosophy, 91(1):139-164, 2013.

[44] Richard Routley. Dialectical logic, semantics and metamathematics. Erkenntnis, 14:301-331, 1979.

[45] Richard Routley and Robert K. Meyer. Dialectical logic, classical logic, and the consistency of the world. Studies in East European Thought, 16(1-2):1-25, 1976.

[46] Stewart Shapiro. Simple truth, contradiction, and consistency. In Graham Priest, Jc Beall, and B. Armour-Garb, editors, The Law of NonContradiction. Oxford University Press, 2004.

[47] Gila Sher. The Bounds of Logic: A Generalized Viewpoint. MIT Press, 1991.

[48] Gila Sher. Tarski's thesis. In Douglas Patterson, editor, New Essays on Tarski and Philosophy, pages 300-339. Oxford University Press, 2008.

[49] Bas C. van Fraassen. Singular terms, truth-value gaps, and free logic. Journal of Philosophy, 63:481-495, 1966.

Australasian Journal of Logic (14:1) 2017, Article no. 1 
[50] Albert Visser. Four valued semantics and the liar. Journal of Philosophical Logic, 13:181-212, 1984.

[51] Zach Weber. A paraconsistent model of vagueness. Mind, 119(476):1026-45, 2010.

[52] Zach Weber, David Ripley, Graham Priest, Dominic Hyde, and Mark Colyvan. Tolerating gluts. Mind, 123(491):813-828, 2014.

[53] P. Woodruff. Paradox, truth, and logic. Journal of Philosophical Logic, 13:213-252, 1984.

Australasian Journal of Logic (14:1) 2017, Article no. 1 